Meta

Journal des tradlucteurs

Translators' Journal

\title{
Le personnel hôtelier
}

\section{Ray J. Pollet}

Volume 23, numéro 2, juin 1978

URI : https://id.erudit.org/iderudit/003851ar

DOI : https://doi.org/10.7202/003851ar

Aller au sommaire du numéro

Éditeur(s)

Les Presses de l'Université de Montréal

\section{ISSN}

0026-0452 (imprimé)

1492-1421 (numérique)

Découvrir la revue

Citer cet article

Pollet, R. J. (1978). Le personnel hôtelier. Meta, 23(2), 167-173.

https://doi.org/10.7202/003851ar d'utilisation que vous pouvez consulter en ligne.

https://apropos.erudit.org/fr/usagers/politique-dutilisation/ 


\section{LE PERSONNEL HÔTELIER}

1. Accountant

2. Accountant's cashier

3. Accounting clerk

4. Assistant accountant

5. Assistant auditor

6. Assistant baker

7. Assistant breakfast cook

8. Assistant captain waiter

9. Assistant convention manager

10. Assistant gardemanger

11. Assistant general manager

12. Assistant head butcher

13. Assistant housekeeper

14. Assistant roast cook

15. Assistant sauce cook

16. Assistant storekeeper

17. Assistant vegetable cook

18. Auditor

19. Baker

20. Baker's helper

21. Banquet manager

22. Barmaid

23. Barroom staff

24. Bartender ; barman

25. Bartender's helper

26. Bellboy ; bellhop

27. Boner
$-\mathrm{A}-$

Comptable

Caissier au Service de la comptabilité

Commis comptable

Comptable adjoint

Aide-vérificateur

Aide-boulanger

Aide-cuisinier préposé aux petits déjeuners

Adjoint au garçon de table en chef

Directeur adjoint au Service des congrès

Préposé adjoint aux buffets froids

Directeur général adjoint

Adjoint au boucher en chef

Aide-ménagère

Aide-rôtisseur

Aide-saucier

Aide-magasinier

Aide-cuisinier préposé aux légumes

Vérificateur

$-\mathrm{B}-$

Boulanger

Garçon boulanger ;

mitron

Directeur du Service des banquets

Barmaid

Personnel de bar

Barman

Commis de bar

Chasseur ;

groom

Désosseur 
28. Brasserie attendant

29. Breakfast cook

30. Buffet chef

31. Busboy

32. Butcher

33. Butcher's helper

34. Captain waiter

35. Captain waitress

36. Carhop ; car attendant

37. Carpenter

38. Carver ; cutter ; slicer

39. Car washer

40. Cashier

41. Caterer

42. Catering manager

43. Cellarman

44. Checker (laundry)

45. Checkroom attendant

46. Chief cook

47. Cleaner

48. Clerical staff

49. Coffeeman

50. Collar finisher (laundry)

51. Confectionery and pastry cook

52. Confectionery chef

53. Convention manager

54. Convention staff

55. Cook

56. Cook's helper ; kitchen hand

57. Cutter (see: Carver)

58. Delivery boy

59. Dishwasher

60. Doorman
Préposé à la brasserie

Cuisinier préposé aux petits déjeuners

Chef préposé au buffet (chaud ou froid)

Débarrasseur ; commis débarrasseur

Boucher

Garçon boucher

$-\mathrm{C}-$

Garçon de table en chef

Serveuse en chef

Garçon chauffeur ; chauffeur placier

Menuisier

Trancheur ; découpeur

Làveur d'automobiles

Caissier/caissière

Traiteur

Chef des approvisionnements

Caviste

Marqueur/marqueuse (buanderie)

Préposé(e) au vestiaire

Chef cuisinier

Nettoyeur

Personnel de bureau

Préposé au café; commis cafetier

Préposé(e) à la finition des cols (buanderie)

Pâtissier-confiseur

Chef confiseur

Directeur du Service des congrès

Personnel préposé aux congrẹ̀s

Cuisinier

Marmiton

- D -

Garçon livreur

Laveur de vaisselle ; plongeur

Portier 
61. Electrician

62. Electrician's helper

63. Elevator mechanic

64. Elevator operator

65. Engineer

66. Entremetier

67. Equipment storeman

68. Errand boy ; messenger boy

69. Fast food cook (see : Short order cook)

70. Fast order cook (see : Short order cook)

71. Fireman

72. First butcher

73. First pastry cook

74. First seamstress

75. First swing cook

76. Flatwork operator

77. Floor pantryman

78. Floor service staff

79. Flower attendant

80. Food and bar cashier

81. Food controller

82. Front office; front desk

83. Front office cashier

84. Fry cook

85. Garage attendant

86. Garage cashier

87. Garage mechanic

88. Garage supervisor

89. Garbage attendant; refuse collector; sanitary engineer

90. Gardemanger

91. General clerk

92. General manager

93. Grillroom cook
$-\mathrm{E}$

Electricien

Aide-électricien

Mécanicien d'ascenseur

Garçon d'ascenseur ;

liftier/liftière

Ingénieur

Entremétier

Préposé à l'équipement

Garçon de courses ; coursier

$-\mathrm{F}-$

Chauffeur (chaudières)

Premier boucher

Premier pâtissier

Première couturière

Premier cuisinier de relève

Repasseur/repasseuse (à la machine)

Préposé à l'office d'étage

Personnel du Service des étages

Préposé(e) aux arrangements floraux; fleuriste

Caissier/caissière (aliments et boissons)

Contrôleur aux vivres

Réception

Caissier/caissière à la réception

Friturier

- $\mathrm{G}-$

Employé de garage

Caissier/caissière de garage

Mécanicien attaché au garage

Surveillant de garage

Préposé aux poubelles

Préposé aux buffets froids

Commis général

Directeur général

Cuisinier préposé aux grillades 
94. Hand ironer

95. Handyman; utilityman

96. Head baker

97. Head bartender

98. Head bellboy; head bellhop

99. Head busboy

100. Head butcher

101. Head carpenter

102. Head cellarman

103. Head fry cook

104. Head gardemanger

105. Head housekeeper

106. Head inspector

107. Head linenkeeper

108. Head maître d'hôtel head maître D

109. Head painter

110. Head pantryman

111. Head payroll clerk

112. Head porter

113. Head roast cook

114. Head sauce cook

115. Head scrubber

116. Head stillroom girl

117. Head swing cook

118. Head upholsterer

119. Head vegetable peeler

120. Head washman (laundry)

121. Housekeeper

122. House nurse

123. House officer; house detective

124. House physician; house doctor

125. Iceman

126. Inspector

127. Ironer
$-\mathrm{H}-$

Repasseur/repasseuse (à la main)

Homme à tout faire

Premier barman

Premier chasseur ; premier groom

Premier débarrasseur; commis débarrasseur principal

Boucher en chef

Chef menuisier

Premier caviste

Friturier en chef

Chef du Service de buffets froids

Chef des services ménagers

Chef inspecteur

Lingère en chef

Premier maître d'hôtel ; chef de brigade

Chef peintre

Chef de la dépense; chef de l'office

Commis principal aux feuilles de paie

Premier porteur

Rôtisseur en chef

Saucier en chef

Premier récureur

Préposée en chef à l'office.

Cuisinier de relève en chef

Chef rembourreur

Chef éplucheur de légumes

Premier laveur (buanderie)

Ménagère

Infirmière d'hôtel ; infirmière attachée à l'hôtel

Détective d'hôtel

Médecin d'hôtel ; médecin attaché à l'hôtel

- I-

Préposé à la glace

Inspecteur

Repasseur/repasseuse 
128. Janitorial staff

129. Kitchen chef

130. Kitchen fireman

131. Kitchen foreman

132. Kitchen hand (see : Cook's helper)

133. Kitchen helper

134. Kitchen steward

135. Laborer ; workman

136. Laundry forelady

137. Laundryman (see: Washman)

138. Liftman (see : Elevator operator)

139. Linenkeeper

140. Lobbyman

141. Locksmith

142. Maid

143. Mail clerk; mail girl

144. Maître d'hôtel ; maître D

145. Manager

146. Messenger boy (see : Errand boy)

147. Night cleaner

148. Night manager

149. Oiler $-\dot{j}-$

Personnel du Service de nettoyage et d'entretien

$-\mathbf{K}-$

Chef des cuisines

Préposé aux fourneaux

Chef de cuisine

Garçon de cuisine

Commis de cuisine

$-\mathbf{L}-$

Journalier ; homme de peine

Buandière en chef

Lingère

Préposé à la salle d'attente

Serrurier

$-\mathrm{M}-$

Servante ;

bonne

Préposé(e) au courrier

Maître d'hôtel ; chef de brigade adjoint

Directeur ; gérant

$-\mathrm{N}-$

Nettoyeur de nuit

Gérant de permanence

$-\mathrm{O}-$

Graisseur 
150. Painter

151. Pantry

152. Pantryman

153. Pastry chef

154. Payroll clerk

155. Plasterer

156. Plumber

157. Plumber's helper

158. Polisher

159. Porter

160. Potwasher

(see: Dishwasher)

161. Press operator

162. Quick service cook (see : Short order cook)

163. Receiving clerk

164. Receptionist

165. Refuse collector (see: Garbage attendant)

166. Relief clerk

167. Relief staff

168. Restroom attendant

169. Roast cook

170. Room maid

171. Room service staff

172. Sauce cook

173. Sanitary engineer (see: Garbage attendant)

174. Scrubber

175. Seamstress

176. Senior accountant

177. Senior payroll clerk

178. Short order cook; fast order cook; fast food cook; quick service cook
$-\mathrm{P}-$

Peintre

Office ; dépense

Préposé à la dépense

Chef pâtissier

Commis aux feuilles de paie

Plâtrier

Plombier

Aide-plombier

Polisseur

Porteur

Presseur/presseuse

$-\mathrm{Q}-$

- R -

Commis à la réception

Réceptionniste

Commis suppléant ; commis de relève

Personnel suppléant; personnel de relève

Préposé(e) aux toilettes

Rôtisseur

Femme de chambre

Personnel du Service des chambres

$-\mathrm{S}-$

Saucier

\author{
Récureur/récureuse \\ Couturière \\ Premier comptable \\ Premier commis aux feuilles de paie \\ Cuisinier préposé aux casse-croûte ; \\ cuisinier de plats-minute; \\ cuisinier de comptoir (cafétérias)
}


179. Slicer

(see : Carver)

180. Soft drink attendant; soda fountain keeper

181. Staff waiter

182. Staff waitress

183. Stenographer

184. Steward

185. Stillroom girl

186. Stillroom staff

187. Storekeeper

188. Swing cook

189. Switchboard operator

190. Telephone operator

191. Tinsmith

192. Uniform room girl

193. Upholsterer

194. Utilityman

(see: Handyman)

195. Valet

196. Valet service attendant

197. Vegetable cook

198. Vegetable peeler

199. Waiter

200. Waitress

201. Wall washer

202. Washman (laundry); laundryman

203. Washman's helper (laundry)

204. Wine steward

205. Workman

(see : Laborer)
Limonadier/limonadière

Garçon de table préposé au personnel

Serveuse préposée au personnel

Sténographe

Préposé au service d'intendance

Préposée à l'office

Personnel de l'office

Magasinier

Cuisinier de relève

Standardiste

- T -

Téléphoniste

Ferblantier

$-\mathrm{U}-$

Préposée aux uniformes

Rembourreur

\section{$-\mathrm{V}-$}

Valet de chambre

Préposé au pressage et au nettoyage

Cuisinier préposé aux légumes

Eplucheur de légumes

- W-

Garçon de table

Serveuse ;

fille de salle

Laveur de murs

Laveur (buanderie)

Aide-laveur (buanderie)

Sommelier 